\title{
Evidence for depinning of a Wigner crystal in $\mathrm{Hg}-\mathrm{Cd}-\mathrm{Te}$
}

\author{
Stuart B. Field, D. H. Reich, B. S. Shivaram, and T. F. Rosenbaum \\ James Franck Institute and Department of Physics, University of Chicago, Chicago, Illinois 60637
}

D. A. Nelson

Electro-Optics Division, Honeywell, Inc., 2 Forbes Road, Lexington, Massachusetts 02172

P. B. Littlewood

AT\&T Bell Laboratories, Murray Hill, New Jersey 07974

(Received 15 April 1985; revised manuscript received 18 July 1985)

\begin{abstract}
We report measurements of the nonlinear current-voltage characteristics on low-carrierconcentration $n$-type samples of $\mathrm{Hg}_{0.76} \mathrm{Cd}_{0.24} \mathrm{Te}$ at high magnetic fields and low temperatures. We find the onset of nonlinear conductivity occurs at applied electric fields of less than $1 \mathrm{mV} / \mathrm{cm}$. The effect can be easily distinguished from sample heating and is consistent with collective transport produced by the sliding of a Wigner crystal pinned by disorder. We estimate a disorder-induced correlation length of tens of micrometers.
\end{abstract}

Narrow-band-gap semiconductors such as $\mathrm{Hg}_{1-x} \mathrm{Cd}_{x} \mathrm{Te}$ are good materials in which to study the localizing effects of magnetic fields. On account of the light conductionband effective mass $m^{*}$ and high dielectric constant $\epsilon_{\infty}$, the effective Bohr radius $a^{*}=\epsilon_{\infty}\left(m / m^{*}\right) a_{B}$ can be as large as $1000 \AA$. Consequently $n$-type samples of $\mathrm{Hg}_{1-x} \mathrm{Cd}_{x} \mathrm{Te}$ show metallic conductivity at millikelvin temperatures and zero magnetic field for carrier densities as low as $10^{14} \mathrm{~cm}^{-3}$. Moderate magnetic fields are a very large perturbation on such a system. For a material with Cd concentration $x=0.24$, such as we study here, the conduction electrons are confined to the lowest spinpolarized Landau level for magnetic fields $H$ exceeding 2 kOe; at higher fields, the transverse overlap of electron wave functions is controlled by the magnetic length $l=(\hbar c / e H)^{1 / 2}$. At a field of only $10 \mathrm{kOe}$, one has $\left(l / a^{*}\right) \simeq 0.3$ and the magnetic field sets the dominant short length scale.

The localization of free-particle wave functions by the magnetic field would be expected to lead to dramatic changes in the conductivity. Indeed, measurements at liquid-helium temperatures ${ }^{1-4}$ have shown that the magnetoresistance and Hall resistance increase abruptly by several orders of magnitude over a narrow range of field centered on a critical field $H_{c}$ which, at a few millikelvin, is a function of temperature. ${ }^{5}$ However, there remains considerable controversy over whether this transition is best described by disorder-induced localization ${ }^{6}$ (which is frequently referred to as "magnetic freeze-out," although this is strictly a concept concerning single-particle binding by isolated donors) or whether electron-electron interactions dominate and the transition is to a charge-density wave $(\mathrm{CDW})$ or Wigner crystal. ${ }^{2,5} \mathrm{~A}$ recent alternative suggestion is that the results may be explained by a combination of bulk localization and extended edge states. ${ }^{7}$

Disorder is of course antagonistic to the formation of a Wigner crystal phase; in three dimensions one expects that there will be no true long-range order even if the disordering potential is weak. Nevertheless, if the effects of disorder are small the crystalline order should persist up to some correlation length $L$, which may be much longer than the lattice spacing $n^{-1 / 3}$. With increasing disorder the correlation length shrinks; once $n^{1 / 3} L \sim 1$ the ground state is better described as an "electron glass.",

Even when the electrons are ordered, fluctuations in the potential will pin the electron lattice. Weak pinning of the Wigner lattice would be expected to lead to a small but finite threshold electric field for nonlinear collective conduction ("sliding") by the lattice. This effect is in direct analogy with the threshold field for sliding of a pinned charge-density wave ${ }^{9}$ in materials such as $\mathrm{NbSe}_{3}$, and can be used to estimate the correlation length $L$. Accordingly, we have searched for nonlinear $I-V$ characteristics in the insulating state at high magnetic fields and low temperatures.

Conventional four-probe resistance measurements were made on $n$-type samples of $\mathrm{Hg}_{0.76} \mathrm{Cd}_{0.24} \mathrm{Te}$ with a carrier density of $1.40 \times 10^{14} \mathrm{~cm}^{-3}$ similar to those used by us in an earlier study. ${ }^{5}$ The samples were oriented with their length along the magnetic field direction and cooled to $T=10 \mathrm{mK}$ in a dilution refrigerator. The small signal levels at which nonlinear $I-V$ effects were observed necessitated a lock-in technique at $37 \mathrm{~Hz}$. All measurements were made in the frequency-independent dc limiting regime.

We plot in the inset to Fig. 1 the $I-V$ characteristics of a sample driven deep into the insulating state by magnetic field $\boldsymbol{H}$. We find a small but well-defined deviation from Ohmic behavior at an rms voltage $V_{d} \simeq 8.6 \times 10^{-5} \mathrm{~V}$, which corresponds to an electric field $\left|\mathbf{E}_{d}\right|=4.9 \times 10^{-4}$ $\mathrm{V} / \mathrm{cm}$. The nonlinear onset can be seen more clearly in the main part of Fig. 1, where we have subtracted out the Ohmic portion $V / R_{0}$ such that deviations from zero represent non-Ohmic character. The nonlinear region is 


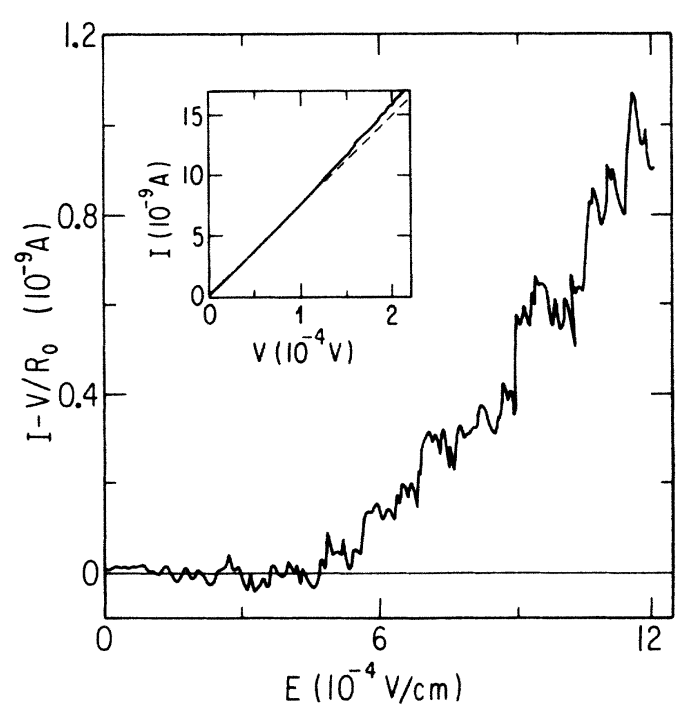

FIG. 1. Nonlinear rms current-voltage characteristic at a field of $64 \mathrm{kOe}$ and a temperature of $10 \mathrm{mK}$. The inset shows the raw data; in the main figure the linear conductivity is subtracted.

accompanied by a large increase in the noise, similar to results ${ }^{9}$ on charge-density-wave compounds. The increase in the noise does not coincide exactly with $\mathbf{E}_{d}$ because these measurements are performed ac; hence, the nonlinear regime is sampled even while the rms voltage is less than $V_{d}$.

Non-Ohmic behavior may arise from simple $I^{2} R$ heating. We rule out heating as the cause for the behavior illustrated in Fig. 1 by investigating, for different values of $H$, the power dissipation $\left(I^{2} R\right)$ associated with the onset of nonlinear effects at $T=100 \mathrm{mK}$. Above $8 \mathrm{kOe}$ in Fig. 2 the threshold power for non-Ohmic behavior drops over 3 orders of magnitude, marking the crossover from simple heating to what we believe is the depinning of a Wigner crystal. The magnetic field at which the crossover occurs is just $10 \%$ above the $H_{c}$ we have previously identified ${ }^{5}$ from Hall-effect data as the critical field for the transition into a Wigner crystal ground state. As expected, we also observe a second region of non-Ohmic behavior for $H>H_{c}$, which is simply the same $I^{2} R$ heating seen for $H<H_{c}$. The decrease (with $H$ ) of the power required to produce Joule heating results because the temperature coefficient of resistance increases with magnetic field (dashed line in Fig. 2). The higher temperature in Fig. 2 ( $T=100 \mathrm{mK}$ ) was chosen because of the superior cooling power of the refrigerator, although the nonlinear behavior is less pronounced at 10 times the $T$ of Fig. 1 .

Figure 3 shows the values of the field $E_{d}$ at which we observe the departure from linear conductivity as a function of magnetic field for two tempertures $T=10$ and 100 $\mathrm{mK}$. The arrows indicate the magnetic fields $H_{c}(T)$, which we determined independently from the linear Hall resistance as marking the phase boundary between the metallic state and a possible Wigner crystal phase. The solid lines are a guide to the eye but it is clear that $E_{d}$ initially decreases as the magnetic field is increased above the transition and then rises slowly with further increase in field.

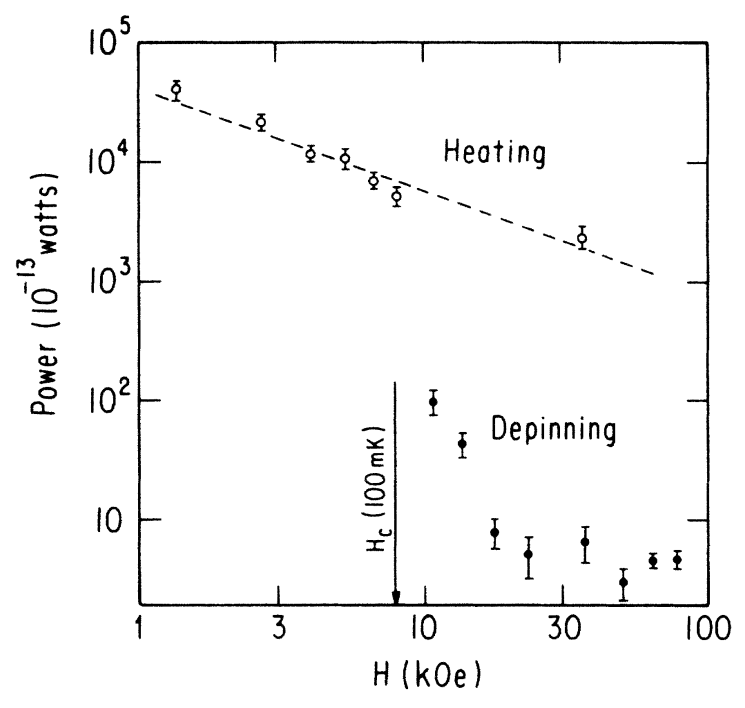

FIG. 2. Power levels associated with the onset of nonlinear resistivity at $T=100 \mathrm{mK}$. The open circles are associated with sample heating; the solid circles correspond to the fields $E_{d}$ shown in Figs. 1 and 3.

Within the error bars this increase is linear. At a temperature of $200 \mathrm{mK}$ we can still observe a nonlinear conductivity at high magnetic fields but not close to $H_{c}$; by $300 \mathrm{mK}$ this effect is unobservable. At a magnetic field of $64 \mathrm{kOe}$ the nonlinear part of the conductivity decreases from a value of $6 \times 10^{-4}(\Omega \mathrm{cm})^{-1}$ at $T=10 \mathrm{mK}$ to $2 \times 10^{-4}(\Omega \mathrm{cm})^{-1}$ at $T=100 \mathrm{mK}$. Over the same region of temperature the linear conductivity is increasing and the nonlinear part becomes a progressively smaller fraction of the whole with increasing temperature.

We argue against impact ionization of electrons as an alternative explanation on several counts. At pumped helium temperatures and zero magnetic field, impact ionization has been observed ${ }^{10}$ in similar stoichiometry $\mathrm{Hg}_{1-x} \mathrm{Cd}_{x} \mathrm{Te}$ at threshold electric fields of $15 \mathrm{~V} / \mathrm{cm}$.

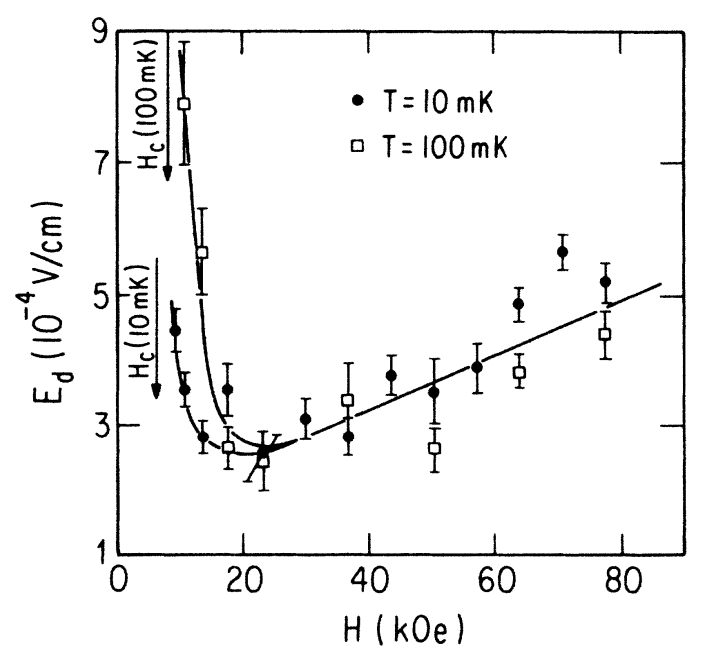

FIG. 3. Magnetic field dependence of the threshold field $E_{d}$ for two temperatures. The solid lines are guides to the eye. 
Here we measure an $E_{d}$ almost 5 orders of magnitude smaller. If the lower temperature and high magnetic field acted to lower the threshold field because shallow donors would now be bound, then $E_{d}$ should rapidly increase with temperature, in contradiction to Fig. 3 . The magnetic field dependence of $E_{d}$ is also difficult to explain from this point of view. Moreover, impact ionization is frequently signaled by a region of negative differential resistance, which we do not observe.

The systematic behavior we observe of the variation of $E_{d}$ with magnetic field and temperature is consistent with a simple model for the collective sliding of a Wigner crystal in an electric field. If we assume that only longwavelength distortions of the Wigner lattice pin the crystal, we may take over the analysis previously applied to sliding CDW systems. ${ }^{11}$ The characteristic length scale $L$ of the lattice distortions is determined by a competition between the elastic stiffness of the lattice and the local random potential. Similar to the case of CDW's we write down a classical model Hamiltonian to describe the interaction of the electron lattice with the underlying potential $\Phi$, valid for $L \gg R_{0} \sim n^{-1 / 3}$;

$H=\int d^{3} r\left(\frac{1}{2} K|\nabla u|^{2}+\rho_{c} E u+\sum_{i} \Phi_{i}\left[r-u(r)-R_{i}\right]\right)$.

Here $u(r)$ is the local distortion of the electron lattice, $K \sim e^{2} R_{0}^{-4} \epsilon_{\infty}^{-1}$ the shear modulus of the lattice, and $\Phi_{i}$ the potential due to impurities at positions $R_{i}$ or other disorder. If $L$ is large compared to the average impurity spacing $c^{-1 / 3}$ ( $c$ is the impurity concentration), then we estimate

$$
K\left(R_{0} / L\right)^{2} \simeq\left(c\left\langle\Phi^{2}\right\rangle / L^{3}\right)^{1 / 2},
$$

where the angular brackets denote a spatial average. The electric field $E$ couples to the full collective charge density $\rho_{c} \simeq e n$; thus the threshold field for sliding will be approximately

$$
E_{T} \simeq A\left(K / \rho_{c} R_{0}\right)\left(L / R_{0}\right)^{-2},
$$

where $A$ is a numerical prefactor of order unity. If we identify $E_{T}$ with the field $E_{d}$ in Fig. 3 we obtain a value of $L / R_{0} \sim 200$ at $H=64 \mathrm{kOe}$ and $T=10 \mathrm{mk}$; i.e., a correlation length of tens of microns. This estimate does not require any assumptions about the strength of the potential $\Phi$; however, if the impurity potential were to dominate we would have $L / R_{0} \simeq 1$. In this strong pinning limit, the electrons are correlated strongly with the impurity potential, and hardly at all with each other; this situation is usually called "magnetic freeze-out."

Magnetic freeze-out would normally be expected for donor impurities interacting with electrons via the Coulomb interaction. In this case, one has $\left\langle\Phi^{2}\right\rangle^{1 / 2} \sim e^{2} \epsilon_{\infty}^{-1}\left(a^{*} l^{2}\right)^{-1 / 3}$, which leads to $\left(L / R_{0}\right)<1$ for magnetic fields exceeding $10 \mathrm{kOe}$. Field ionization of electrons bound to donors would then be expected to occur at electric fields $\sim 100 \mathrm{~V} \mathrm{~cm}^{-1}$, although impact ionization should preempt this effect at somewhat lower electric fields, but certainly much larger than the observed $E_{d}$. The absence of magnetic freeze-out in our samples may be explained by a large reduction in $\Phi(r)$ from two effects. Firstly, it is believed that there is a large repulsive "central-cell" correction, which leads to donor levels resonant with the conduction band at least in small magnetic fields. ${ }^{12}$ Secondly, the (attractive) Coulomb tail of the donor potential will be strongly disrupted by shortrange disorder. A reduction in the value of $\left\langle\Phi^{2}\right\rangle^{1 / 2}$ by roughly a factor of 20 from the Coulomb result (at $H \sim 50$ kOe) would be consistent with our estimate of the correlation length $L$.

The increase in the threshold field at large magnetic fields is to be expected because the electronic wave function is shrinking, and therefore the electron will "see" more of the random potential. Typically, one would estimate that $\Phi$ scales with magnetic length as $l^{-2 / 3}$ (as, for example, is the case for an electron bound in a Coulomb well). Equations (1) and (2) then lead to $E_{T} \sim l^{-8 / 3} \sim H^{4 / 3}$ at high magnetic fields, which is consistent with our data within the error bars and corresponds to $L$ decreasing as $H^{-2 / 3}$. At magnetic fields close to the onset of the crystalline phase, one expects that the elastic modulus $K$ is reduced by fluctuations, and since $E_{T} \sim K^{-3}$ this would account for the rise in $E_{T}$ as the magnetic field is decreased in the vicinity of $H_{c}$. One also expects $E_{T}$ to increase with temperature in this region, as we also observe. We note that a rise in $E_{T}$ close to the CDW onset temperature is frequently observed in sliding CDW compounds. ${ }^{9}$

The value of the dc threshold $E_{d}$ combined with the slope of the nonlinear region allows us to estimate a characteristic relaxation frequency in the kilohertz range for the linear ac response of a pinned Wigner lattice. Broad band noise in the same frequency range is to be expected when the lattice is sliding.

While all the data presented here are consistent with our interpretation of a magnetic field induced Wigner crystallization, there are some features for which we do not have a complete explanation. We note that even at the lowest measuring temperature $(10 \mathrm{mK})$ the nonlinear conductivity is only $10 \%$ of the measured linear conductivity. This ratio of the nonlinear-to-linear conductivity is not to be taken as a measure of the "condensate" fraction however, because the relaxation mechanisms for the two processes may be quite different. We also find the sharp rise in $E_{d}$ as the magnetic field is lowered through $H_{c}$ to be surprisingly large within the context of a presumed firstorder transition. This indicates very strong "premelting" phenomena with a reduction in the shear modulus of about $30 \%$ near $H_{c}$ at $T=100 \mathrm{mK}$.

In summary, we have used a magnetic field to drive low-electron density metallic samples of $\mathrm{Hg}_{0.76} \mathrm{Cd}_{0.24} \mathrm{Te}$ through the metal-insulator transition and have observed nonlinear $I-V$ behavior which allows us to characterize the nature of the insulating ground state. Our data are consistent with the existence of a Wigner crystal with a domain size of tens of microns. The sliding of the Wigner crystal allows us to probe its structure, in analogy with measurements on CDW compounds, ${ }^{9}$ and recent work on graphite in high magnetic fields. ${ }^{13}$ 
We would like to thank R. M. Fleming, A. Libchaber, S. R. Nagel, G. A. Thomas, and C. M. Varma for helpful discussions. The work at the University of Chicago was supported by the National Science Foundation (NSF) under Grant No. DMR-83-05065. D.H.R. received support from NSF Grant No. DMR-83-51992. T.F.R. would also like to thank the Alfred P. Sloan Foundation for financial assistance.
${ }^{1}$ G. de Vos and F. Herlach, in Application of High Magnetic Fields in Semiconductor Physics, Vol. 177 of Springer Lecture Notes in Physics, edited by G. Landwehr (Springer, Berlin, 1983), p. 378.

${ }^{2} \mathrm{G}$. Nimtz and B. Schlicht, in Festkörperprobleme $X X$, edited by J. Treusch (Vieweg, Braunschweig, 1980), p. 360.

${ }^{3}$ A Raymond, J. L. Robert, R. L. Aulombard, C. Bousquet, and O. Valassiades, in Physics of Narrow Gap Semiconductors, Vol. 152 of Springer Lecture Notes in Physics, edited by E. Gornik, H. Heinrich, and L. Palmetshofer (Springer, Berlin, 1982), p. 387.

${ }^{4}$ M. Shayegan, H. D. Drew, D. A. Nelson, and P. M. Tedrow Phys. Rev. B 31, 6123 (1985).

${ }^{5}$ T. F. Rosenbaum, S. B. Field, D. A. Nelson, and P. B. Littlewood, Phys. Rev. Lett. 54, 241 (1985).

6J. L. Robert, in Narrow Gap Semiconductors: Physics and Applications, Vol. 133 of Springer Lecture Notes in Physics, edited by W. Zawadski (Springer, Berlin, 1980), p. 176.
${ }^{7}$ M. Ya. Azbel, Solid State Commun. 54, 127 (1985).

${ }^{8}$ B. T. Shklovskii and A. L. Efros, Zh. Eksp. Teor. Fiz. 64, 2222 (1973) [Sov. Phys.-JETP 37, 1123 (1973)].

${ }^{9}$ For reviews, see G. Grüner, Physica 8D, 1 (1983); N. P. Ong, Can J. Phys. 60, 757 (1982).

${ }^{10}$ V. N. Kobysev and A. S. Tager, Pis'ma Zh. Eksp. Teor. Fiz. 14, 164 (1971) [JETP Lett. 14, 107 (1971)]; G. Nimtz, G. Bauer, R. Dornhaus, and K. M. Muller, Phys. Rev. B 10, 3302 (1974).

${ }^{11}$ H. Fukuyama and P. A. Lee, Phys. Rev. B 17, 535 (1978); P. A. Lee and T. M. Rice, ibid. 19, 3970 (1979); H. Fukuyama and P. A. Lee, ibid. 18, 6245 (1978).

${ }^{12}$ B. Schlicht and G. Nimtz, in Physics of Narrow Gap Semiconductors, Vol. 152 of Springer Lecture Notes in Physics, edited by E. Gornik, H. Heinrich, and L. Palmetshofer (Springer, Berlin, 1982), p. 383.

${ }^{13}$ Y. Iye and G. Dresselhaus, Phys. Rev. Lett. 54, 1182.(1985). 